\title{
Automated Algorithm for Determining the Interplanar Distances of the Crystal Structure of a Substance from Transmission Electron Microscopy Images
}

\author{
S.G. Nebaba ${ }^{1}$, A.Ya. $\mathrm{Pak}^{2}$, A.A. Zakharova ${ }^{3}$ \\ stepan-lfx@mail.ru|ayapak@tpu.ru|zaa@tu-bryansk.ru \\ ${ }^{1}$ Keldysh Institute of Applied Mathematics RAS, Moscow, Russia; \\ ${ }^{2}$ Tomsk Polytechnic University, Tomsk, Russia; \\ ${ }^{3}$ Bryansk State Technical University, Bryansk, Russia
}

\begin{abstract}
A problem of automated image processing of transmission electron microscopy and its application value is considered in this paper. An automated algorithm for estimating the interplanar distances of the crystal structure of a substance from transmission electron microscopy images is proposed. The software implementation of the algorithm was developed and tested on several raster images, and the evaluation results were compared with the results obtained using the specialized software named Gatan Microscopy Suite v.1.8. The high degree of coincidence of the results showed the viability of the proposed approach and the prospects of its further development in the area of transmission electron microscopy images processing.
\end{abstract}

Keywords: computer vision, image processing, image analysis, transmission electron microscopy.

\section{Introduction}

Computer vision methods and algorithms associated with processing and analysis of raster images are widely used in various fields of science [1,4,5,7,8], including the field of processing of images, obtained by electron microscopes [6]. One of the fundamental problems in these areas is the automation of materials composition and structure evaluation according to their images. Effective solution of this problem simplifies the tasks of non-destructive quality control of materials and products, their identification and determination of their properties and appearance [6].

The development of information technology and computing devices contributes to rapid progress in solving such type of problems. However, to date, existing methods of identification and evaluation of micro-objects in the raster image do not have sufficient versatility, which would allow them to be easily automated. In addition, these techniques, methods and algorithms are often part of proprietary software, which is closely associated with electron microscopy equipment and is protected by copyright of manufacturers of this equipment [2]. The cost of such equipment can reach hundreds of millions of rubles, which is unacceptable for a sufficiently significant part of researchers and scientific organizations.

At the same time, systems for analyzing such images can be widely demanded by many scientific organizations as well as by enterprises in the manufacturing sector, where they can be applied for the on-line monitoring and analysis of the composition and structure of materials and products from them. Cheap analogues of existing software for critical areas of enterprise activities can stimulate the development of technologies for the synthesis, analysis and production of micro and nanomaterials. Finally, improving the characteristics of materials for various purposes and products based on them can have a positive effect on the level of safety in many areas of human activity as well as promote the development of new technologies.

The practical application of specialized software for image processing obtained with an electron microscope can be seen in [6] and many others papers. This fact indicates that the field of knowledge is developing in the modern world.

Thus, the considered interdisciplinary problem seems relevant and promising. There is a need in the systematization of the existing methods and algorithms for automated evaluation and image processing and in the development of software for evaluating and analyzing electron microscopy images on their basis. In the future, this can contribute to the development of software systems for image analysis of electron microscopes with the possibility of mass use and implementation.

\section{Automated algorithm for estimating the width of the layers of the material in a raster image}

The key idea of the proposed algorithm is based on selection, normalization, and evaluation of a part of the raster image obtained using an electron microscope, which has a regular layered structure.

The algorithm can be described with the following sequence of actions:

1. The user selects the area of the image that needs to be analyzed. It is enough to select 4 points, each of which is inside a regular structure in the image.

2. The program rotates the image at a certain angle through affine transformations in order to build a regular structure strictly perpendicular to the $\mathrm{x}$-axis and simplify further calculations [3].

3. The image is binarized with a given threshold, which allows to split the pixels, defining the layers through white pixels, and the gaps between the layers through black pixels [8]

4. The sequence of sums of white pixels along the $x$-axis is calculated, which shows the pixel density.

5. After calculation of the first derivative of the sequence obtained in Section 4, a sequence of values characterizing the number of transitions between layers can be obtained. Positive values of the derivative characterize the proximity to the center of the layer, negative ones characterize the proximity to the center of the gap between the layers.

6. After determination of the extreme right and extreme left local maxima of the sequence, as well as the first local minimum, the threshold for the intersection of the $\mathrm{x}$-axis can be set and, after counting the number of such intersections between the maxima, the average evaluation of the layer width, which is measured in the number of points of the original image, can be calculated.

7. The width of the layer, which is estimated by the physical quantity, can be obtained with the knowledge about the ratio between image points and real physical quantities.

In general, the formula for calculating the average layer width in the image can be represented as follows:

$$
L_{s}=\frac{L_{N}}{n * L_{i}},
$$

where $L_{s}$ is the width of one layer, measured by a physical quantity (for example, $\mathrm{nm}$ ), 
$L_{N}$ is the length of the selected area with a regular structure of the material in the image, in pixels,

$n$ is the number of layers in the selected area, calculated using the proposed algorithm,

$L_{i}$ is the ratio of the image size in pixels with real values (for example, pixels / $\mathrm{nm}$ ).

The proposed algorithm is quite simple in terms of implementation and does not require large computational resources, but in suggested form it requires a number of manual actions from the user: in particular, such actions as selecting the area of the analyzed structure in the image, checking the correctness of the rotation angle, choosing the binarization threshold and image ratios with real physical quantities are predominantly manual at this moment. Almost all of these limitations can be easily overcome by choosing an appropriate way for parameters selection and preparation of images for analysis, but the initial choice of the analysis area is always left to the software user.

The described algorithm has been tested on several high resolution TEM-images (Transmission electron microscopy images) taken in the direct resolution mode, when crystalline planes are visible in the composition of the crystalline material directly in the image (Fig. 1).

\section{Software implementation of the algorithm and testing on real data}

The software implementation of the proposed algorithm was performed in the Microsoft Visual Studio development environment using the open source library of computer vision algorithms and image processing openCV.

Figure 1 presents examples of the original images, with which the proposed algorithm was tested.

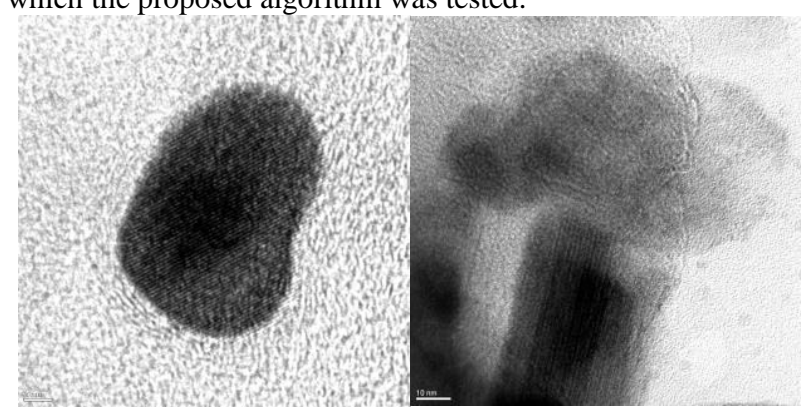

Fig. 1. Examples of TEM-images shoot in direct resolution mode.

Figure 2 shows the results of rotation and binarization of selected areas of the images.

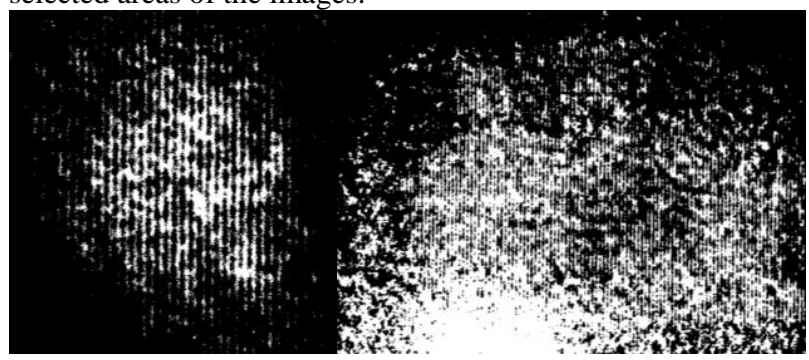

Fig. 2. Examples of images, which were binarized and rotated in the direction of the viewed planes of the crystal structure perpendicular to the $\mathrm{x}$-axis.

Figure 3 shows the sequences characterizing the density distribution of white pixels of images along the $\mathrm{x}$-axis.

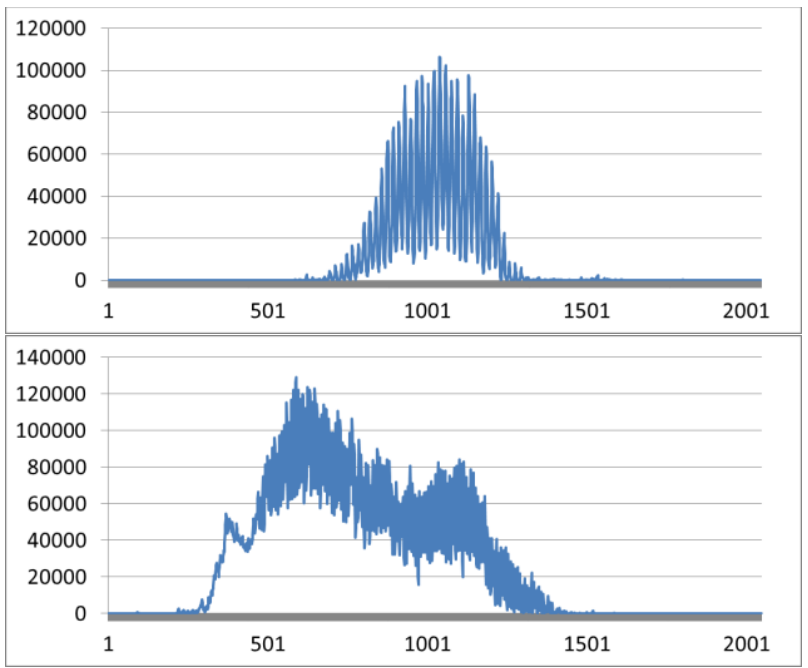

Fig. 3. Graphs of pixel density in binarized and rotated images.

Figure 4 shows the derivatives of the sequences, which represent the number of "layers" (atomic planes) in the image.

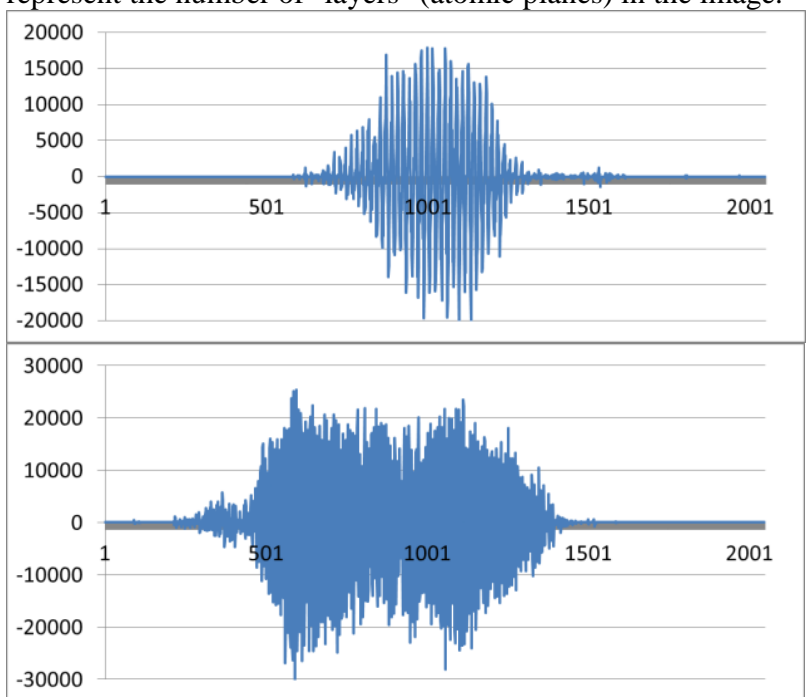

Fig. 4. Derivatives of the pixel density sequences.

Table 1 presents parameters of the algorithm with which the tests were carried out, as well as the final result of the evaluation of the average layer width in the image, calculated by formula (1).

Table 1. The parameters of the algorithm and the result of evaluation the width of the layer in the image

\begin{tabular}{|c|c|c|}
\hline Parameter name & Image 1 & Image 2 \\
\hline Binarization threshold & $5 / 255$ & $100 / 255$ \\
\hline Angle of rotation & 62 & 41 \\
\hline $\begin{array}{c}\text { Range of points (by X- } \\
\text { axis) }\end{array}$ & $763-1216$ & $505-799$ \\
\hline $\mathrm{L}_{\mathrm{i}, \text { pixels / nm }}$ & 74 & 19 \\
\hline $\begin{array}{c}\text { The number of layers in } \\
\text { the range, } \mathrm{n}\end{array}$ & 25 & 40 \\
\hline $\begin{array}{c}\text { Estimation of the } \\
\text { average interplanar } \\
\text { distance, nm }\end{array}$ & 0.2449 & 0.3868 \\
\hline
\end{tabular}

The obtained results were compared with the results of calculations of specialized software supplied as part of an analytical complex based on a JEOL JEM $2100 \mathrm{~F}$ transmission electron microscope (Gatan Microscopy Suite v.1.8) [2]. 


\section{Comparison of the results of the proposed algorithm with the results of specialized software}

Image 1 processing with the Gatan Microscopy Suite v.1.8 software (Fig. 5) made it possible to determine the interplanar spacing using the Process FFT function as part of an integrated assessment of the entire particle (image 1), which shows a single crystal, and the resulting interplanar spacing, $\mathrm{d}=0.2468$ $\mathrm{nm}$, was obtained. At the same time, the Profile function made 5 measurements of interplanar distances in different parts of the crystal. According to the results of these measurements, the value $\mathrm{d}=0.2498 \mathrm{~nm} \pm 0.0033 \mathrm{~nm}$ was determined.

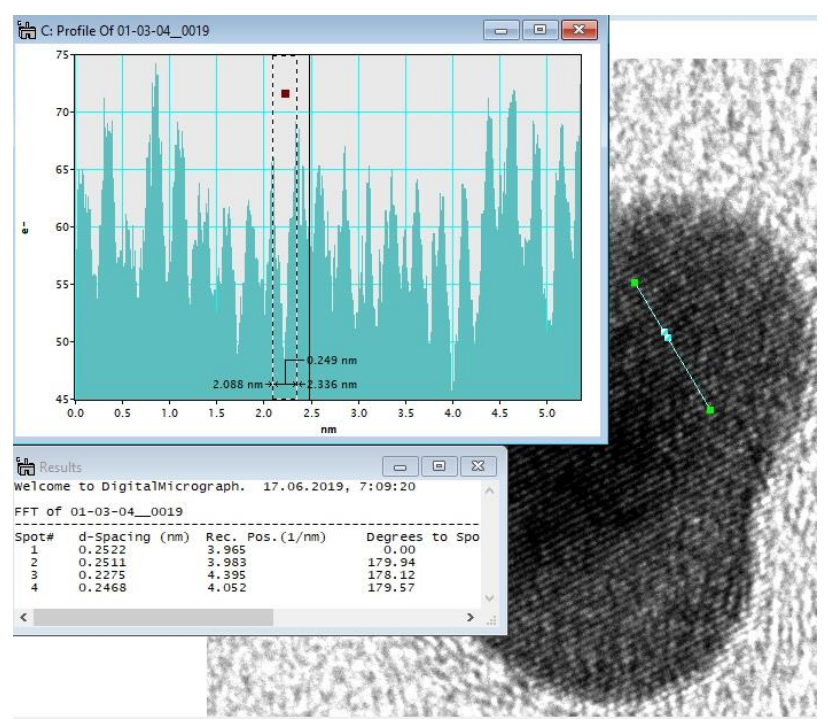

Fig. 5. The result of image 1 processing with the Gatan Microscopy Suite v.1.8 software.

It is impossible to conduct an integral evaluation using the Process FFT function for image 2 due to the presence of several types of crystalline objects in the image. However, the Profile function makes it possible to select the same part of the analyzed image area 2, which was analyzed above by the author's algorithm. Figure 6 shows the software window during image processing.

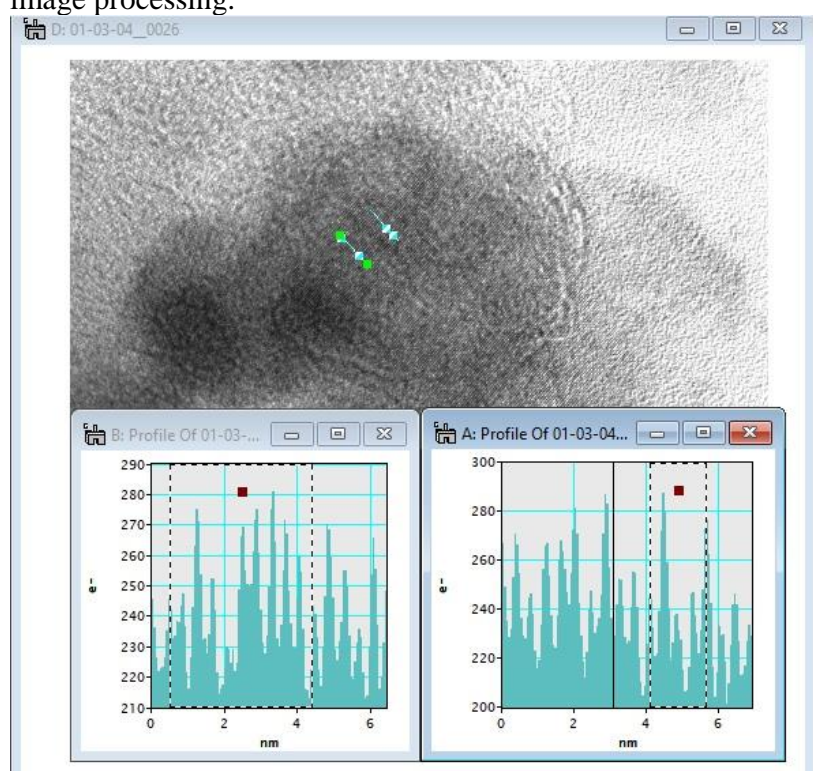

Fig. 6. The result of image 2 processing with the Gatan Microscopy Suite v.1.8 software.
The value of the interplanar distance $d=0.3870 \mathrm{~nm} \pm 0.012$ $\mathrm{nm}$ was determined as a result of measurements. Lower image quality, as well as a smaller zoom coefficient can be the reasons of the larger deviation in this case.

As a result of comparing the values obtained by the developed algorithm with the values of specialized software used as reference, it can be concluded that there are insignificant variances in the obtained data. Differences of data, which were by the developed algorithm, from the "reference" data are about $0.005 \mathrm{~nm}$. Also it should be noted that the specialized software package Gatan Microscopy Suite v.1.8 uses in its work a "raw" image file with total data volume equals 16.9 MB, saved directly during the operation of the electron microscope, and usually accessible to operators of electron microscopes. At the same time, the developed algorithm analyzes the standard graphics file with JPEG extension and a data volume less than $1 \mathrm{MB}$, which, as a rule, is the best quality that generally available to persons conducting image analysis. Thus, the ability to work directly with the public JPEG file is shown in this work.

\section{Conclusion}

The paper reviewed the existing approach to processing and analyzing images of the crystal structure of substances using specialized software for analysis of transmission electron microscopy data.

The analysis of the problem of evaluation the average width of the interplanar distance in the image is carried out; image processing methods suitable for the task of automating the calculation of the interplanar spacing of the crystal structure of a substance, which is shown in images taken in the direct resolution mode, are highlighted.

An algorithm that allows to calculate in a partially automatic mode the interplanar distance of the crystal structure of a substance is proposed. The proposed algorithm was tested on several images, the numerical results were compared with the results obtained with the same images using specialized software that designed for analysis of images obtained with using a transmission electron microscope.

The use and further development of the proposed image processing algorithm will make it possible to solve the problem of evaluation of the average value of the interplanar distance in the image without the involvement of specialized software. It makes sense to improve the proposed algorithm both in the direction of better automatization of the evaluation process and accuracy increasing. In addition, in the future, it seems appropriate to consider the possibility of analyzing local areas in the image in order to search for anomalies in the crystal cell, or, in other words, its defects.

\section{Acknowledgments}

This work has been supported the Ministry of Education and Science of the Russian Federation by the Grant No. 2.1642.2017/4.6.

\section{References}

[1] Alhadidi B. Mammogram Breast Cancer Image Detection Using Image Processing Functions / B. Alhadidi, M.H. Zu'bi, H.N. Suleiman. // Information Technology Journal. 2007. Vol. 6. №. 2. P. 217-221.

[2] Gatan Microscopy Suite Software [Electronic Source]. URL: https://www.gatan.com/products/tem-analysis/gatanmicroscopy-suite-software. (Last accessed: 11.06.2019).

[3] Gonzalez R.C. Digital Image Processing (3rd Edition) / R.C. Gonzalez, R.E. Woods // Prentice-Hall, Inc., Upper Saddle River, NJ, USA, 2006. P. 976. 
[4] Leutenegger S. BRISK: Binary Robust invariant scalable keypoints / S. Leutenegger, M.Chli, R.Y. Siegwart // Proceedings of the 2011 International Conference on Computer Vision (ICCV '11). 6 November 2011. P. 25482555.

[5] Nebaba S.G. An Algorithm for Building Deformable 3d Human Face Models and Justification of its Applicability for Recognition Systems / S.G. Nebaba, A.A. Zakharova // SPIIRAS Proceedings. 2017. Vol. 52. P. 157-179.

[6] Pak A. Synthesis of ultrafine cubic tungsten carbide in a discharge plasma jet / A. Pak, A. Sivkov, I. Shanenkov, I. Rahmatullin, K. Shatrova // International Journal of Refractory Metals and Hard Materials. 2015. Vol. 48. P. 51-55. ISSN 0263-4368. https://doi.org/10.1016/j.ijrmhm.2014.07.025.

[7] Schettini R. Underwater Image Processing: State of the Art of Restoration and Image Enhancement Methods / R. Schettini, S. Corchs // EURASIP Journal on Advances in Signal Processing. 2010.

[8] Sokratis V. A Hybrid Binarization Technique for Document Images / V. Sokratis, E. Kavallieratou, R. Paredes, K. Sotiropoulos // In: Biba M., Xhafa F. (eds) Learning Structure and Schemas from Documents. Studies in Computational Intelligence. 2011. Vol. 375. Springer, Berlin, Heidelberg. 